\title{
BMJ Early closure of temporary Open ileostomy-the EASY trial: protocol for a randomised controlled trial
}

To cite: Danielsen AK, Correa-Marinez A, Angenete $\mathrm{E}$, et al. Early closure of temporary ileostomy-the EASY trial: protocol for a randomised controlled trial. BMJ Open 2011:1:e000162.

doi:10.1136

bmjopen-2011-000162

- Prepublication history for this paper is available online. To view these files please visit the journal online (http:// bmjopen.bmj.com).

Received 5 May 2011

Accepted 24 June 2011

This final article is available for use under the terms of the Creative Commons Attribution Non-Commercial 2.0 Licence; see http://bmjopen.bmj.com

${ }^{1}$ Department of Surgery, Herlev Hospital, University of Copenhagen, Herlev, Denmark

${ }^{2}$ Department of Surgery, Sahlgrenska University Hospital/Östra, Göteborg, Sweden

${ }^{3}$ Department of Surgery, Karnsjukhuset, Skovde, Sweden

Correspondence to Anne Kjaergaard Danielsen; adan0009@heh.regionh.dk

\author{
Anne Kjaergaard Danielsen, ${ }^{1}$ Adiela Correa-Marinez, ${ }^{2}$ Eva Angenete, ${ }^{2}$ \\ Stefan Skullmann, ${ }^{3}$ Eva Haglind, ${ }^{2}$ Jacob Rosenberg, ${ }^{1}$ SSORG (Scandinavian \\ Outcomes Research Group)
}

\begin{abstract}
Objective: The objective is to evaluate efficiency based on data on morbidity and mortality, health-related quality of life and healthcare-related costs after early reversal of temporary ileostomy after rectal resection for cancer compared with the standard procedure (late reversal).
\end{abstract}

Background: Reversal of a temporary ileostomy is generally associated with a low morbidity and mortality. However, ostomy reversal may cause complications requiring reoperation with subsequent major complications, in ranges from $0 \%$ to $7-9 \%$ and minor complications varying from $4-5 \%$ to $30 \%$.

Based on studies exploring and describing the time of closure in previous studies which are mostly of low quality, a recent review concluded that closing a temporary stoma within 2 weeks did not seem to be associated with an increase in morbidity and mortality. Design and methods: Early closure of temporary ileostomy (EASY), a randomised controlled trial, is a prospective randomised controlled multicentre study which is performed within the framework of the Scandinavian Surgical Outcomes Research Group (http://www.ssorg.net/) and plans to include 200 patients from Danish and Swedish hospitals. The primary end-point of the study is the frequency of complications $0-12$ months after surgery (the stoma creation operation). The secondary end-points of the study are (1) comparison of the total costs of the two groups at 6 and 12 months after surgery (stoma creation); (2) comparison of health-related quality of life in the two groups evaluated with the 36-item shortform and European Organization for Research and Treatment of Cancer Quality of Life QuestionnaireCR29/CR30 at 3, 6 and 12 months after surgery (stoma creation); and (3) comparison of diseasespecific quality of life in the two groups at 3,6 and 12 months after surgery (stoma creation).

Discussion: The aim of the EASY trial is to evaluate the efficiency of early reversal of temporary ileostomy after surgery for rectal cancer versus late reversal. The EASY trial is expected to have a huge impact on patient safety as well as an improvement in patient-reported outcome.

Clinical trials identifier: NCT01287637.

\section{ARTICLE SUMMARY}

\section{Article focus}

Frequency of complications $0-12$ months after initial surgery; comparison of health-related quality of life in the two groups evaluated with the 36-item short-form and European Organization for Research and Treatment of Cancer Quality of Life Questionnaire-CR29/CR30 at 3, 6, and 12 months after surgery (stoma creation); and comparison of the total costs of the two groups at 6 and 12 months after the initial surgery (stoma creation).

\section{Key messages}

The significance of the study is the aim of making evidence-based recommendations for timing of the closure of a temporary ileostomy after surgery for rectal cancer.

Strengths and limitations of the study The dimensions of the study (sample size: 200 patients) allow us to make recommendations. The recommendations will be of central importance to future patients. The results not only incorporate complications and mortality but integrate patientreported outcome. The limitation is that the followup period is limited to 12 months.

\section{INTRODUCTION}

Intestinal anastomoses are frequently created in patients undergoing surgery for rectal cancer with simultaneous construction of a diverting ileostomy. ${ }^{1}$ This is done to limit the consequences of anastomotic leakage. A recent Cochrane review concluded that a temporary ileostomy is associated with fewer anastomotic leakages, ${ }^{2}$ and a decrease in the need for urgent reoperation has also been reported. ${ }^{3}$

Reversal of a temporary ileostomy is generally associated with a low mortality. ${ }^{4}$ However, ostomy reversal may cause complications requiring reoperation, and a recently published review found major complications ranging from $0 \%$ to $7-9 \%$ and minor 
complications varying from $4-5 \%$ to $30 \% .^{5}$ Based on studies exploring and describing the time of closure, the authors suggested that closing a temporary stoma within 2 weeks did not seem to be associated with an increase in morbidity and mortality, and might even reduce the complication rate. The article referred to a relatively small number of studies which were mostly of low scientific quality, and recommended future randomised, prospective, large-scale studies. ${ }^{4}$

Stoma creation affects patients differently, and the reactions cover perceptions of an altered body image, changes in daily routines, lifestyle and sexuality. ${ }^{6-8}$ On the other hand, stoma creation is a treatment that eliminates disease, relieves pain and improves health, whereby stoma creation may also have a positive impact. ${ }^{9}$ Several individual factors are related to the individual's adaptation to life with a stoma including age, ${ }^{10}$ socioeconomic profile, ${ }^{11}$ personality ${ }^{12}$ and sex. ${ }^{13}$ Studies have shown inferior quality of life in patients with a stoma compared with those who underwent similar procedures without stoma formation. ${ }^{14-18}$ Reversal of the temporary stoma resulted in an increase in quality of life, ${ }^{14}$ whereas knowing that the situation was temporary could interfere with adaptation to living with a temporary ileostomy. ${ }^{19}$

Measurements of health-related quality of life are increasingly demanded in the healthcare sector ${ }^{2021}$ and are proposed as one suitable test of efficiency of clinical interventions. ${ }^{22}$ Studies measuring other parameters following stoma reversal, instead of focusing solely on terms of morbidity and mortality and disregarding patient-centred outcomes, are therefore warranted.

Efficacy can also be connected to health economic evaluations. It is essential to describe the economic effect when exploring early reversal of ileostomies, as the healthcare sector is met with constant demands of justifying any developments in existing treatments. ${ }^{23} \mathrm{An}$ economic efficacy analysis is, therefore, a way to support a professional process of prioritising, when introducing new treatments and methods. ${ }^{24}$

These findings all point to the obvious: this issue requires further investigation in a randomised clinical trial in order to be able to make reliable recommendations. The time for reversal of the stoma is an issue of central importance, and we therefore aim to investigate morbidity and mortality, health economic implications as well as patient-reported outcome related to the time of reversal of a temporary ileostomy after surgery for rectal cancer.

\section{METHODS}

\section{Study design}

Early closure of temporary ileostomy (EASY), a randomised controlled trial, is a prospective randomised controlled multicentre study which is performed within the framework of the Scandinavian Surgical Outcomes Research Group (http://www.ssorg.net) and plans to include 200 patients from Danish and Swedish hospitals (figures 1, 2).
The objective is to evaluate efficiency based on data on morbidity and mortality, health-related quality of life and healthcare-related costs after early reversal of temporary ileostomy compared with standard procedure (late reversal).

Patients in the intervention group will have the stoma reversed 8-13 days after stoma creation, and patients in the control group will have the stoma reversed after a minimum of 12 weeks. Patient enrolment started in February 2011 and is expected to end in February 2012 with a total of 200 patients included. The study is performed in compliance with the ethical principles of the World Medical Association Declaration of Helsinki, as well as the Danish and Swedish data-protection agencies and ethical committees. Patients receive complete oral and written information about the trial, and a written consent has to be signed. The study protocol has been registered at http://www.clinicaltrials. gov/ (identifier: NCT01287637).

\section{Study population}

Patients eligible for enrolment into EASY are 18+ years of age and have all received surgical treatment (low anterior resection of the rectum) for rectal cancer with the construction of a temporary ileostomy. Adjuvant therapy will be registered in the case report form, and is not an exclusion criterion.

During the first days after surgery, the patients undergo an individual assessment and screening process describing their postsurgical state. The assessment is carried out by a surgeon, and the patients are categorised and scored regarding bowel function, functional level of nutrition and mobilisation. The surgeon performs a physical examination 2-3 days after surgery and states whether or not faeces and gas have passed from the ileostomy, how the patient is nourished and how many hours the patient is out of bed. This assessment is carried out before scanning of the anastomosis, to prevent the surgeon being biased by the result.

Six to 8 days after creation of the stoma, patients eligible for inclusion will undergo a CT of the rectum with a water-soluble contrast medium to visualise the anastomosis and possible leakage. The contrast medium (eg, Omnipaque, with an iodine concentration of $350 \mathrm{mg} / \mathrm{ml}$, manufactured by GE Healthcare, Waukesha, Wisconsin) is diluted in isotonic saline to a $5 \%$ solution (50 $\mathrm{ml}$ of Omnipaque, with an iodine concentration of $350 \mathrm{mg} / \mathrm{ml}$ in $1000 \mathrm{ml}$ of isotonic saline).

The contrast medium is instilled using a Foley catheter placed in the rectum just below the anastomosis. The catheter must be placed high enough in the rectum to prevent it from sliding out accidentally, as the balloon is not inflated. The infusion of the contrast $(300-500 \mathrm{ml})$ takes about 5-10 min, and patients do not experience any discomfort during this procedure. If there is a leak of contrast outside the rectum, the patient will not be included in the study. 
Figure 1 Flow chart EASY. EORTC, European Organization for Research and Treatment of Cancer; OAS, Ostomy Adjustment Scale; QLQ, Quality of Life Questionnaire;

SF-36, Short Form 36.

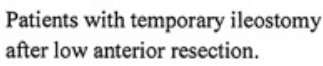

Data on the assessment and the results of the CT scan are entered into a screening log, where information on all patients, entering or not entering the study, is described.

The exclusion criteria are: patients with diabetes, patients being treated with steroids, patients with linguistic difficulties and patients with expected low compliance.

Patients are included if they consent to participate after receiving both oral and written information, and if they meet the inclusion criteria, do not have radiologically identifiable anastomotic leaks and are assessed to be fit to undergo surgery, based on the aforementioned screening and assessment process.

\section{Randomisation}

Patients who have provided written informed consent preceding inclusion and who meet all inclusion criteria and none of the exclusion criteria are randomised to either early or late reversal of the temporary ileostomy. Inclusion and randomisation are carried out shortly after the CT scan.

The randomisation is based on blocks of six. The randomisation list is computer-generated using dedicated software (http:/ /www.randomization.com). The randomisation list is not known to the local or head investigators, as it is kept by an administrative employee, who sends out the needed case report forms and the correlating randomisation numbers. In case of drop-outs, the study will include new blocks of six patients to ensure randomisation and balance between the two groups.
Obviously, neither patients nor surgeons are blinded, and all observations related to treatment are documented in the regular patient file.

\section{Surgical technique}

The surgical technique is the standard procedure, and the closure of the stoma is carried out with either a suture-only or stapling-only technique,${ }^{25}$ depending on the local guidelines.

The surgeon will register information regarding the surgical intervention: ASA score, whether the anastomosis was hand-sutured or stapled, blood loss, duration of surgery and anaesthetic method.

\section{End-points}

The primary end-point of the study is:

- Frequency of complications 0-12 months after initial surgery.

The secondary end-points of the study are:

- Comparison of the total costs of the two groups at 6 and 12 months after the initial surgery (stoma creation);

- Comparison of health-related quality of life in the two groups evaluated with the 36-item short-form and European Organization for Research and Treatment of Cancer Quality of Life Questionnaire-CR29/CR30 at 3, 6 and 12 months after surgery (stoma creation);

- Comparison of disease-specific quality of life in the two groups at 3,6 and 12 months after surgery (stoma creation) as long as the stoma is not yet reversed. All participating centres will register both primary and secondary end-points. 
Figure 2 Tempogram EASY. C-DCSC, Clavien-Dindo

Classification of Surgical Complications; EORTC, European Organization for Research and Treatment of Cancer; OAS, Ostomy Adjustment Scale; QLQ, Quality of Life Questionnaire; QOL, Quality of Life; SF-36, Short Form 36.

\begin{tabular}{|c|c|c|c|c|c|c|c|c|}
\hline & $\begin{array}{l}\text { Day 2-3 } \\
\text { after stoma } \\
\text { creation }\end{array}$ & $\begin{array}{l}\text { Day 6-8 } \\
\text { after stoma } \\
\text { creation }\end{array}$ & $\begin{array}{l}\text { Inclusion } \\
\text { and } \\
\text { randomisat } \\
\text { jon }\end{array}$ & $\begin{array}{l}\text { Day 8-13 } \\
\text { after stoma } \\
\text { creation }\end{array}$ & $\begin{array}{l}3 \text { months } \\
\text { after stoma } \\
\text { creation }\end{array}$ & $\begin{array}{l}\text { Min. 12 } \\
\text { weeks after } \\
\text { stoma } \\
\text { creation }\end{array}$ & $\begin{array}{l}6 \text { months } \\
\text { after stoma } \\
\text { creation }\end{array}$ & $\begin{array}{l}12 \text { months } \\
\text { after stoma } \\
\text { creation }\end{array}$ \\
\hline $\begin{array}{l}\text { Assessment of } \\
\text { postoperative } \\
\text { restitution by } \\
\text { surgeon }\end{array}$ & $\mathbf{x}$ & & & & & & & \\
\hline $\begin{array}{l}\text { CT-scan of the } \\
\text { rectum }\end{array}$ & & $\mathrm{x}$ & & & & & & \\
\hline $\begin{array}{l}\text { Early reversal } \\
\text { of stoma }\end{array}$ & & & & $\mathbf{x}$ & & & & \\
\hline $\begin{array}{l}\text { Late reversal of } \\
\text { stoma }\end{array}$ & & & & & & $\mathbf{x}$ & & \\
\hline $\begin{array}{l}\text { Registration of } \\
\text { complications } \\
\text { C-DCSC }\end{array}$ & & & & & $\mathbf{x}$ & & $\mathbf{x}$ & $\mathbf{x}$ \\
\hline QOL SF-36 & & & & & $\mathbf{x}$ & & $\mathrm{x}$ & $\mathrm{x}$ \\
\hline $\begin{array}{l}\text { QOL EORTC } \\
\text { CR30/29 }\end{array}$ & & & & & $\mathrm{x}$ & & $\mathbf{x}$ & $\mathbf{x}$ \\
\hline $\begin{array}{l}\text { QOL OAS (only } \\
\text { control group } \\
\text { for as long as } \\
\text { the stoma is in } \\
\text { place) }\end{array}$ & & & & & $\mathrm{x}$ & & $\mathrm{x}$ & $\mathbf{x}$ \\
\hline
\end{tabular}

Patient assessment and follow-up

All patients will be monitored closely in relation to complications, health-related quality of life and costs.

\section{Complications}

In order to ensure an objective assessment of complications following surgery, each patient is evaluated according to the Clavien-Dindo Classification of Surgical Complications. ${ }^{26} 27$

The classification score classifies complications according to the necessary treatment, which prevents an individual evaluation of the patient. Whether something is to be classified as a minor or major complication or as 'severe' or 'less severe', is avoided by using the classification. This ensures that data are based on objective criteria, whereby the influence of personal and/or cultural conditions is minimised. There are seven grades (I, II, IIIa, IIIb, IVa, IVb and V), starting with grade I, 'any deviation from the normal postoperative course without the need for pharmacological treatment or surgical, endoscopic and radiological interventions' and ending with grade $\mathrm{V}$, 'death of a patient'. In this sense, mortality, tied to the surgical intervention, is classified as the most severe complication of all.

The classification has been used in several clinical studies, and the method has been validated extensively.

All complications are logged, and both the type and severity of each complication are documented at hospital discharge, and 3, 6 and 12 months after surgery.

\section{Health-related quality of life: the instruments}

Short form 36 is a generic tool that evaluates quality of life from patients' self-reports on different states influencing quality of life. It consists of 36 items that measure eight dimensions of health on a multi-item scale. The instrument measures physical functioning, social functioning and role limitations because of physical and emotional problems. The scoring scale ranges from 0 to 100 , with lower scores indicating worse health. The instrument has been extensively validated, and baseline data in both Danish ${ }^{28}$ and Swedish ${ }^{29}$ populations have been published.

The Ostomy Adjustment Scale is a disease-specific questionnaire that measures the patients' subjective adaptation to life with a stoma. The focus is on physical, mental as well as social modifications following stoma creation. The questionnaire has 34 questions and is widely used and validated within different cohorts of patients with stomas. Scoring ranges from 1 to 6 , and lower scores indicate a lower quality of life. ${ }^{30} 31$

European Organization for Research and Treatment of Cancer CR30 is a cancer-specific questionnaire of five functional scales (physical, role, cognitive, emotional and social), three symptom scales (fatigue, pain and nausea, and vomiting), one global health status and quality-of-life scale, and six single-item measures (dyspnoea, insomnia, appetite loss, constipation, diarrhoea and financial difficulties). A high score for a functional scale represents a high level of functioning, whereas a high score for a symptom scale represents a high level of symptoms.

The CR29 is a questionnaire designed for use in patients undergoing treatment for colorectal cancer. It consists of questions assessing disease symptoms, sideeffects of treatment, body image, sexuality and future perspective. Both questionnaires together consist in total of 59 questions and are validated internationally and available in Danish and Swedish versions. ${ }^{32} 33$

Each questionnaire is applied at 3,6 and 12 months after stoma creation, and apart from the Ostomy Adjustment Scale, which will only be administered to the control group as long as patients have a stoma, each included patient will be assessed at all times.

\section{Cost measurement}

Costs are measured across the patients' course, which includes length of hospital stay, visits to outpatient clinics and interventions in the primary care setting. 
Data from the hospital stay are electronically logged at each hospital at the time when they occur. Data from the primary care sector are self-reported data, and patients fill out a patient-diary with check-box options. Furthermore, patients report prospectively on social activities, as this information is hard to access retrospectively. ${ }^{34} 35$

Health-related economic assessments are done 3, 6 and 12 months after the initial operation (including stoma creation).

\section{Sample size}

The sample size estimation is based on data from a randomised trial with a similar case-mix, although complications were not described in detail and did not discern the severity of the complication. ${ }^{36}$ This study showed a complication rate of $38 \%$ in patients, whose ileostomy was reverted later. With the differences in describing the main outcome in mind, we propose a conservative assumption of $30 \%$ complications in the control group and $10 \%$ in the intervention group, a power of $80 \%$ and a significance level of $5 \%$. This gives a needed total sample of 144 patients (72 in each group). We have chosen to include 100 patients in each group.

\section{Statistical analysis}

Regarding the primary end-point, the two groups will be compared using the $\chi^{2}$ test. Furthermore, a CI for the difference between the two proportions will also be calculated, using a Gaussian approximation. For comparing the cost assessment between the two groups, a Student $t$ test will be used, or a Welsh version of the test in case of differing variance. If the distribution is significantly skewed, the Mann-Whitney test will be used instead. For analysing quality of life, non-parametric tests will be used in all analyses-that is, we will use the Mann-Whitney test, Wilcoxon test and $\chi^{2}$ test, where relevant. Variations over time within the same patient group will be analysed with the Friedman analysis of variance. Data will be described using medians and ranges. We plan to carry out an intention-to-treat analysis as well as a per-protocol analysis. Generally, $\mathrm{p}<0.05$ will be considered statistically significant. If randomisation fails with generating comparable groups, that is, if the two groups differ significantly at the start, for example, country, gender and age, we will use multivariate approaches in order to adjust for these differences.

\section{DISCUSSION}

EASY is a randomised clinical trial in patients with rectal cancer and explores the efficiency of early reversal (8-13 days) of a temporary ileostomy created simultaneously with a low anterior resection compared with the standard reversal, which is usually after 3 months.

A randomised and controlled study with 186 patients reversed the stoma 8-10 days after surgery compared with the standard procedure, where the stoma was reversed after $62-69$ days. ${ }^{36}$ In this study, there were no significant differences in frequencies of complications
(15\% in both groups), but there were significant differences in types of complications, where the early closure group had significantly more wound complications, and the late closure group had significantly more cases of small-bowel obstruction. ${ }^{36}$

A prospective study showed that earlier reversal (median 11 days instead of 2-3 months) was not associated with increased morbidity or mortality. ${ }^{37} \mathrm{~A}$ small randomised study investigated the role of early closure (10 days after surgery) of ileostomies in 36 preoperatively selected patients. ${ }^{38}$ This study found that the length of hospital stay was significantly shorter in the intervention group and that the time until bowel function and resumption of oral nutrition did not differ between the groups. Thus, the authors concluded that the early intervention was not associated with increased risk of complications. ${ }^{38}$

A review has shown that outcome did not differ significantly between early and late reversal of a covering ileostomy regarding morbidity and mortality. ${ }^{5}$ Based on the findings of studies exploring the timing of the reversal, it was concluded that if early reversal is chosen, reversal of the temporary stoma should preferably be done within 14 days from the creation of the stoma. ${ }^{5}$

Although complications are generally a reported parameter, the severity of the complications and the implications for the patients have often not been described sufficiently. Moreover, a literature review stated that definitions of complications such as bowel obstruction, ileus and wound sepsis varied between papers, ${ }^{4}$ which was supported by another study that found similar problems when comparing complication frequencies. ${ }^{39}$ The study reported high frequencies of complications as well as a remarkably high mortality ranging from $1 \%$ (temporary colostomy) to $5.3 \%$ (temporary ileostomy) when reversing the stomas. ${ }^{39}$ Deaths were related to anastomotic leaks, sepsis, acute myocardial infarction and one death of unknown cause.

Use of the Clavien-Dindo Classification of Surgical Complications will improve the identification and classification of complications to type and severity, as it will reduce subjective interpretation and any tendency to downrate complications. ${ }^{26}$ Its main focus is on the necessary treatment of the complication, which is a marker for the severity. The term 'wound complication' covers major as well as minor levels of severity, and as such does not offer a precise description. Moreover, The Clavien-Dindo Classification of Surgical Complications seems to be influenced by the level of experience with the medical staff using it, ${ }^{40}$ and it is recommended that the assessment be carried out by committed personnel, which in this study is achieved by engaging specialist professionals as local investigators.

A variety of questionnaires or tools have been used to assess quality of life as it pertains to specific health outcomes. In the healthcare sector, the counterpart of quality-of-life measures are health-outcome measures other than biomedical. These may include an ability to 
perform tasks of daily living, energy level and other indicators of well-being associated with, but not identical to, the medical condition.

When measuring health-related quality of life, some studies have failed to use a precise and sufficient tool, as questionnaires may have to be combined in order to obtain valid data. ${ }^{20} 2841$ Other studies have focused on health-related quality-of-life measurements that solely monitor gastrointestinal symptoms, which might explain difficulties in the interpretation. ${ }^{7} 12144243$ Although some of the findings have been inconsistent, the main results were that patients with colorectal cancer both with and without a stoma reported limitations to their quality of life, but patients with a stoma generally reported more impairment than patients without a stoma. ${ }^{15}$ However, a low anterior resection with routine temporary loop ileostomy produced a marked impairment in health-related quality of life in the months after construction, and improved after ileostomy closure. ${ }^{44}$

In designing a trial that undertakes socio-economic measurements alongside the medical and health-related data, we wished to obtain, as far as possible, a real-life design. Although all trials present challenges to those undertaking socio-economic measurements, these are methodologically lessened in the case of more realistic trials. $^{45} 46$

For this reason, the inclusion criteria are based on everyday surgical decision-making, which includes and combines extensive surgical experience of the individual surgeon with the systematic stratification model offered. Besides that, all patients eligible for inclusion, but not included, will be logged in order to present a detailed description with clinically relevant real-life data.

The economic data include costs related to hospital care and out-of-hospital care, for instance in the stoma clinic. However, in order to present data that supersede the hospital-based care and treatment, this trial focuses on costs generated from visits to the general practitioner, primary care as well as costs related to patients' individual socio-economic data. This entails, for instance, patient-reported time-data covering returning to work, and socially relevant data indicating cancer patients' adaptation to living with a stoma versus living without a stoma. ${ }^{34} 47$

In conclusion, we have designed a multi-centre study, in order to answer a relatively unexplored and complex scientific question within everyday surgery, the timing of the reversal of a temporary ileostomy created to prevent leakage after low anterior resection due to rectal cancer. Earlier studies have shown that the timing is a central element regarding morbidity and mortality, but so far only a few studies have explored the additional issues such as the psychosocial and economic impact of early versus late reversal of stomas with a follow-up period of 12 months. With the dimensions of the EASY trial, we hope to be able to make evidence-based recommendations for timing of the closure of a temporary ileostomy after surgery for rectal cancer.
Competing interests None.

Ethics approval Ethics approval was provided by the committees on biomedical research ethics of the capital region of Denmark.

Provenance and peer review Not commissioned; externally peer reviewed

Data sharing statement No additional data available.

\section{REFERENCES}

1. Chand M, Nash GF, Talbot RW. Timely closure of loop ileostomy following anterior resection for rectal cancer. Eur J Cancer Care (Engl) 2008;17:611-15.

2. Montedori A, Cirocchi R, Farinella E, et al. Covering ileo- or colostomy in anterior resection for rectal carcinoma. Cochrane Database Syst Rev 2010;(5):CD006878.

3. Matthiesen P, Hallböök O, Rutegaard J, et al. Defunctioning stoma reduces symptomatic anastomotic leakage after low anterior resection of the rectum for cancer. Ann Surg 2007;246:207-14.

4. Chow A, Tilney HS, Paraskeva $P$, et al. The morbidity surrounding reversal of defunctioning ileostomies: a systematic review of 48 studies including 6,107 cases. Int J Colorectal Dis 2009; 24:711-23.

5. Hindenburg T, Rosenberg J. Closing a temporary ileostomy within two weeks. Dan Med Bul 2010;57:1-5.

6. Brown H, Randle J. Living with a stoma: a review of the literature. J Clin Nurs 2005;14:74-81.

7. Gooszen AW, Geelkerken RH, Hermans J, et al. Quality of life with a temporary stoma: ileostomy vs. colostomy. Dis Colon Rectum 2000;43:650-5.

8. Nugent KP, Daniels $P$, Stewart B, et al. Quality of life in stoma patients. Dis Colon Rectum 1999;42:1569-74.

9. Rauch P, Miny J, Conroy T, et al. Quality of life among disease-free survivors of rectal cancer. J Clin Oncol 2004;22:354-60.

10. Ma N, Harvey J, Stewart J, et al. The effect of age on the quality of life of patients living with stomas: a pilot study. ANZ J Surg 2007;77:883-5.

11. Holzer B, Matzel K, Schiedeck T, et al. Do geographic and educational factors influence the quality of life in rectal cancer patients with a permanent colostomy? Dis Colon Rectum 2005;48:2209-16.

12. Siassi M, Hohenberger W, Lösel F, et al. Quality of life and patient's expectations after closure of a temporary stoma. Int $J$ Colorectal Dis 2008;23:1207-12.

13. Hamashima C. Long-term quality of life of postoperative rectal cancer patients. J Gastroenterol Hepatol 2002;17:571-6.

14. Engel J, Kerr L. Quality of life in rectal cancer patients. A four-year prospective study. Ann Surg 2010;238:203-13.

15. Sprangers MA, Taal BG, Aaronson NK, et al. Quality of life in colorectal cancer. Stoma vs. nonstoma patients. Dis Colon Rectum 1995;38:361-9.

16. Butler DL. Early postoperative complications following ostomy surgery: a review. J Wound Ostomy Continence Nurs 2009;36:513-19.

17. Cottam J, Richards K, Hasted A, et al. Results of a nationwide prospective audit of stoma complications within 3 weeks of surgery. Colorectal Dis 2007:9:834-8.

18. Shabbir J, Britton DC. Stoma complications: a literature overview. Colorectal Dis 2010;12:958-64.

19. Smith DM, Loewenstein G, Jankovic A, et al. Happily hopeless: adaptation to a permanent, but not to a temporary, disability. Health Psychol 2009;28:787-91.

20. Gronvold M. Health-related quality of life in cancer patients (in Danish). Ugeskr Laeger 2008;170:838-43.

21. Jess P. Quality of life assessments in colorectal surgery (in Danish). Ugeskr Laeger 2008;170:853-5.

22. Burckhardt CS, Hanestad BR. Nursing strategies and quality of life outcomes: a systematic review. Vard i Norden 2003;1:4-9.

23. Wittrup-Jensen KU, Lauridsen J, Pedersen KM. Ønsker danskerne mest mulig sundhed for pengene, når de prioriterer? In: Lauridsen JT, Pedersen KM, eds. Sundhedsøkonomi. Fra Teori til Praksis. Copenhagen, Denmark: Jurist-og Økonomforbundet, 2009:161-74.

24. van den Hout WB, van den Brink M, Stiggelbout AM, et al. Costeffectiveness analysis of colorectal cancer treatments. Eur $J$ Cancer 2002;38:953-63.

25. Horisberger K, Beldi G, Candinas D. Loop ileostomy closure: comparison of cost effectiveness between suture and stapler. World $\mathrm{J}$ Surg 2010;34:2867-71.

26. Dindo D, Demartines N, Clavien PA. Classification of surgical complications: a new proposal with evaluation in a cohort of 6336 patients and results of a survey. Ann Surg 2004;240:205-13. 
27. Dindo D, Clavien PA. What is a surgical complication? World J Surg 2008;32:939-41.

28. Bue Bjoerner J, Trab Damsgaard M, Watt T, et al. Dansk Manual Til SF36. Copenhagen, Denmark: Lif, Lægemiddelindustriforeningen, 1997.

29. Taft C, Karlsson J, Sullivan M. Performance of the Swedish SF-36 version 2.0. Qual Life Res 2004:13:251-6.

30. Burckhardt CS. The Ostomy Adjustment Scale: further evidence of reliability and validity. Rehabil Psychol 1990;35:149-55.

31. Olbrisch M. Development and validation of the Ostomy Adjustment Scale. Rehabil Psychol 1983;28:12.

32. Gujral S, Conroy T, Fleissner C, et al. Assessing quality of life in patients with colorectal cancer: an update of the EORTC quality of life questionnaire. Eur J Cancer 2007;43:1564-73.

33. Whistance RN, Conroy T, Chie W, et al. Clinical and psychometric validation of the EORTC QLQ-CR29 questionnaire module to assess health-related quality of life in patients with colorectal cancer. Eur $J$ Cancer 2009;45:3017-26.

34. Goossens ME, Rutten-van Mölken MP, Vlaeyen JW, et al. The cost diary: a method to measure direct and indirect costs in costeffectiveness research. J Clin Epidemiol 2000;53:688-95.

35. van Berge Henegouwen MT, van Driel HF, Kasteleijn-Nolst Trenite DG. A patient diary as a tool to improve medicine compliance. Pharm World Sci 1999;21:21-4.

36. Alves $\mathrm{A}$, Panis $\mathrm{Y}$, Lelong $\mathrm{B}$, et al. Randomized clinical trial of early versus delayed temporary stoma closure after proctectomy. Br J Surg 2008;95:693-8.

37. Bakx R, Busch OR, van GD, et al. Feasibility of early closure of loop ileostomies: a pilot study. Dis Colon Rectum 2003;46:1680-4.
38. Menegaux F, Jordi-Galais $\mathrm{P}$, Turrin N, et al. Closure of small bowel stomas on postoperative day 10. Eur J Surg 2002; 168:713-15.

39. Nagell CF, Pedersen CR, Gyrtrup HJ. [Complications after stoma closure. A retrospective study of 11 years' experience] (in Danish). Ugeskr Laeger 2005;167:1742-5.

40. Dindo D, Hahnloser D, Clavien PA. Quality assessment in surgery: riding a lame horse. Ann Surg 2010;251:766-71.

41. Gronvold M. [Quality of life research methodology]. Ugeskr Laeger 2008; $170: 825-9$.

42. Camilleri-Brennan J, Steele RJ. Objective assessment of morbidity and quality of life after surgery for low rectal cancer. Colorectal Dis 2002;4:61-6.

43. Neumann HB, Patil S, Fuzesi S, et al. Impact of a temporary stoma on the quality of life of rectal cancer patients undergoing treatment. Ann Surg Oncol 2011;18:1397-403.

44. OLeary DP, Fide CJ, Foy C, et al. Quality of life efter low anterior resection with total mesorectal excision and temporary loop ileostomy for rectal carcinoma. Br J Surg 2001;88:1220.

45. Drummond M. Introducing economic and quality of life measurements into clinical studies. Ann Med 2001;33:344-9.

46. Drummond MF, Sculpher MJ, Torrance GW, et al. Methods for the Economic Evaluation of Health Care Programmes. 3rd edn. Oxford: Oxford University Press, 2005.

47. Kronborg C, Vass M, Lauridsen J, et al. Cost effectiveness of preventive home visits to the elderly. Economic evaluation alongside randomized controlled study. Eur $J$ Health Econ 2006;7:238-46. 